\title{
Healthy Sleepers Can Worsen Their Sleep by Wanting to Do so: The Effects of Intention on Objective and Subjective Sleep Parameters
}

This article was published in the following Dove Press journal: Nature and Science of Sleep

\section{Selina Ladina Combertaldi Björn Rasch (D)}

Division of Cognitive Biopsychology and Methods, Department of Psychology, University of Fribourg, Fribourg, FR, Switzerland
Correspondence: Björn Rasch Division of Cognitive Biopsychology and Methods, Department of Psychology, University of Fribourg, Rue P.-A.-Faucigny

2, Fribourg $\mathrm{CH}-1700$, Switzerland

Tel +4I 263007637

Email bjoern.rasch@unifr.ch
Purpose: Sleep is regulated by homeostatic and circadian factors. In addition, psychological factors have a strong modulatory impact on our sleep, but the exact underlying mechanisms are still largely unknown. Here, we examined the role of intentions on subjective and objective sleep parameters. Young healthy sleepers were instructed to voluntarily either worsen or improve their sleep. We predicted that participants would be capable of worsening, but not improving, their sleep compared to a regular sleep condition. In addition, we predicted that the instruction to alter sleep would lead to a higher discrepancy between subjective and objective sleep variables.

Participants and Methods: Twenty-two healthy students participated in one adaptation and three experimental nights. Polysomnography and subjective sleep parameters were measured during all four nights. Participants were instructed to sleep regularly ("neutral"), better ("good") or worse ("bad") than normal, in a counterbalanced order.

Results: The instruction to sleep "bad" increased objective sleep onset latency and the number of awakings during the night. The effects were stronger on subjective sleep variables, resulting in a higher sleep misperception in the "bad" condition as compared to the other two conditions. The instruction to sleep "good" did not improve sleep nor did it affect sleep misperception.

Conclusion: We conclude that intention is sufficient to impair (but not improve) subjective and objective sleep quality and to increase sleep misperception in healthy young sleepers. Our results have important implications for the understanding of the impact of psychological factors on our sleep.

Keywords: cognition, sleep, sleep quality, intention, sleep misperception

\section{Plain Language Summary}

Sleep is characterized by a reduction in consciousness. Therefore, sleep is seen often as a biological state that cannot be influenced by our intention. Here we study how that healthy young sleepers are capable of objectively worsening (but not improving) their sleep simply by wanting to do so. When participants intended to sleep worse, they indeed reported a lower sleep quality and objectively had a lower sleep efficiency. In addition, participants strongly overestimated their induced sleep disturbances, as typically seen in insomnia patients, and performed worse in a reaction time test in the morning. In contrast, healthy young sleepers were not capable of further improving their sleep by wanting to do so. Our results show that our pre-sleep intentions and goals to manipulate sleep can affect ongoing sleep processes and influence evaluations of our sleep quality. Our results suggest that psychological process continues to be active during the state of sleep and can influence biological sleep regulation and maintenance in spite of the reductions in consciousness. 


\section{Introduction}

Sleep is a natural and reversible state of reduced consciousness which is regulated by complex neurobiological mechanisms and homeostatic processes. ${ }^{1}$ In the case of disturbed sleep, a wide range of different sleep-inducing drugs are available which are known to help fall asleep as well as to maintain it. $^{2-5}$ Based on the increased understanding of sleep-regulating neurobiology, new drugs have been developed and are available to allow a better preservation of the natural sleep architecture, ${ }^{6,7}$ in particular with respect to the restorative stage of slow-wave sleep (SWS). ${ }^{8,9}$

In addition to the neurobiological aspects of sleep, psychological factors play a critical role in sleep onset and maintenance. According to the cognitive models of insomnia, ${ }^{10,11}$ insomnia patients tend to strongly worry about their sleep and about the negative consequences of not getting enough sleep. This worry might increase attention for cues that pose a threat to their sleep and increase nonfunctional safety behaviors. Together with false beliefs about sleep, excessive sleep anxiety is presumably a causal factor for sleep disturbances. In addition, patients with insomnia can have a strong misperception of their actual sleep quality resulting in a large subjective-objective discrepancy of sleep parameters such as sleep onset latency (SOL), number of awakenings from sleep (NWAK) and time spent awake at night (WASO). ${ }^{12}$ According to the hyperarousal model of insomnia, ${ }^{13}$ cognitive-psychological factors act in concert with biological arousal processes to inhibit sleep. Consequently, relaxation of somatic tension and reduction of worries, together with cognitive therapy on sleep beliefs, are key aspects of cognitive-behavioral therapy for insomnia (CBT-I) ${ }^{14}$ CBT-I uses behavioral strategies such as psychoeducation, stimulus control, sleep restriction, and paradoxical intervention. ${ }^{14,15}$

While it seems clear from insomnia research that our mental activity and thoughts can disturb our sleep, the basic mechanisms are not fully understood. Importantly, it is not clear to what extent we can manipulate healthy sleep intentionally. Intentions are a part of our executive functions, mainly involving neuronal networks in the prefrontal cortex, ${ }^{16,17}$ which can have a modulatory top-down influence on the basic neurophysiological functioning of our brain. Sleep, when considered as a state of strongly reduced consciousness, typically appears to be beyond our cognitive control. Therefore, it is important to examine whether, and to what extend, healthy participants can voluntarily influence their sleep. Furthermore, it is highly relevant to explore the possible mechanisms underlying the influence of voluntary intentions on sleep. For example, participants might be able to alter their biological or somatic arousal state voluntarily before sleep, which in turn might affect subsequent sleep. Alternatively, the possible effects of intention on sleep might depend on mental activity per se, independently from somatic pre-sleep arousal. In addition, our intention might influence the discrepancy between subjective and objective sleep parameters often seen in insomnia patients.

To test the effect of intentions on sleep, a number of young healthy participants spent three nights in the sleep laboratory and were instructed to sleep "as bad as possible", "as good as possible" or "as usual", in a counterbalanced order. They were instructed to manipulate sleep latency, the number and duration of awakenings, and sleep depth, according to the respective condition. They received no instructions regarding how they should achieve these manipulations. Sleep was recorded using a polysomnography setup. As we expect participants to be able to manipulate sleep intentionally, we hypothesized that participants would be able to sleep worse when asked to do so, as indicated by subjective and objective sleep parameters. However, because nighttime sleep in young healthy participants is already close to optimal, we do not expect intentions to make sleep better in our study, thus the condition to sleep "as good as possible" will be invariant across sleep parameters compared to the control condition. We also expect that effects of intentions on sleep will be larger for subjective as compared to objective sleep parameters, resulting in a higher subjective-objective sleep discrepancy. Finally, we will examine the association between somatic arousal before and after sleep (as measured by electrocardiography (ECG)) to test whether sleep changes induced by intention could be solely explained by changes in pre-sleep arousal or not.

\section{Participants and Methods Participants}

Twenty-four young healthy students ( 15 women) with a mean age of $22.3 \pm 3.0$ [SD] participated in the study. Two participants were excluded due to the detachment of electrodes during the night. Participants were recruited through an advertisement and a newsletter for students. They were not on any night shifts and were asked to keep a regular sleep rhythm. Participants were no extreme chronotypes for morningness or eveningness. Based on the German translations of the 
Morningness-Eveningness Questionnaire (D-MEQ ${ }^{18}$ ) four participants were moderate morningness and four participants were moderate eveningness types. Participants reported that they did not suffer from any known sleep disorders, however they did report a high subjective sleep quality. (Pittsburgh Sleep Quality Index (PSQI) ${ }^{19} 3.95 \pm 1.68$ [SD]). They were instructed to abstain from alcoholic and caffeinated beverages on experimental days as well as the day before the test days. The local ethics committee of the University of Fribourg approved the study and all subjects gave written informed consent prior to participating. This is in accordance with the declaration of Helsinki. Subjects received either university course credits or financial compensation of at least $180 \mathrm{CHF}$ for participating in the experiment. In case of an early drop out, the payment was provided proportionately.

\section{Experimental Design and Procedures}

The within-subject design included one adaptation and three experimental nights in the sleep laboratory at the University of Fribourg with the different conditions: "bad", "good" and "neutral" sleep. The procedure of the three experimental conditions was identical with the exception of the instructions given to the participants prior to sleep (see below). The experimenter and the subject were blind towards the condition until the very moment before sleeping. The order of the conditions was randomly assigned and balanced between the subjects.
Participants arrived at the sleep lab and filled in some standardized questionnaires before the installation of the polysomnography (PSG including electroencephalogram (EEG), electromyogram (EMG), electrooculogram (EOG), electrocardiogram (ECG)). They then completed a memory task where they learned 40 semantically associated word pairs (paired associated learning (PAL) $\operatorname{task}^{20}$ ) before going to bed, after which they were instructed to lay in bed and the instructions for the night were given to them ("Sleep as well/badly/neutrally as possible"). Eight hours after lights out, the experimenter woke the participants up. Directly after getting up, participants filled in standardized questionnaires (concerning subjective sleep rating, mood), and completed a psychomotor vigilance test (PVT), as well as the morning-recall of the PAL. The session ended with the removal of the PSG (see Figure 1).

\section{Materials}

\section{Instructions}

Instructions were given orally when participants were lying in bed and were ready to sleep. The instructions were as follows:

- "Bad" sleep: participants were instructed to sleep as bad as possible. They should try to fall asleep as late as possible (ideally, they should not fall asleep at all), wake up during the night (and if so, stay awake),

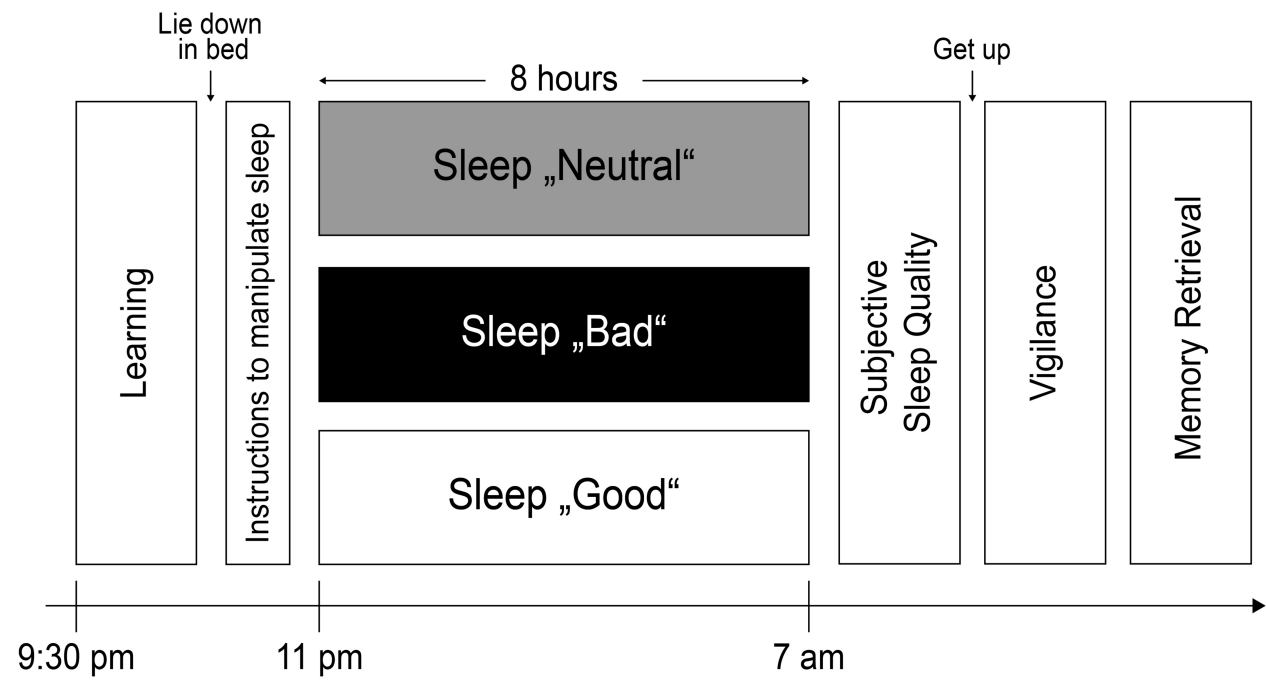

Figure I Procedure and experimental design. Participants spent one adaptation night and three experimental nights in the sleep lab for a total of four nights, each separated by one week. Directly before going to sleep and when lying in bed, participants received the instructions to sleep worse than normal (sleep "bad"), better then normal (sleep "good") or to sleep as usual (sleep "neutral"). In the "bad" condition, they were asked to willingly decrease sleep quality by falling asleep later, waking up more often, staying awake longer during the night and sleeping less deep. In the "good" condition, participants were instructed to decrease sleep quality by falling asleep quicker, staying asleep and sleeping as deeply as possible. No instructions were given regarding how participants could achieve these goals. Participants had to stay in bed in the dark and were not allowed to get up during the night. Sleep was recorded using a polysomnography setup. Before the instructions, participants filled out questionnaires and performed a wordpair learning task. After sleep participants filled out questionnaires, performed a psycho-vigilance task (PVT) and retrieved word-pairs learned before sleep. 
sleep less time, and sleep only lightly (enhance the light sleep).

- "Good" sleep: participants were instructed to sleep as well as possible. They were instructed to fall asleep quickly (ideally directly after the experimenter turned off the light), not wake up during the night (if so, they should try to fall back asleep fast), sleep as long as possible (ideally the full $8 \mathrm{hrs)}$ ) and sleep as deeply as possible.

- "Neutral" sleep: in the neutral night, participants were given the following instructions: "This is your neutral night. Sleep as regularly as possible."

For all three conditions, participants were instructed to stay in bed in the darkness. They were not allowed to get up, read, or access their mobile phones. No additional instructions were given on how to reach "good" vs "bad" sleep. The participants were told that if they were able to sleep better/worse as indicated by the PSG measurements, they would be rewarded with an additional $25 \mathrm{CHF}$ for each experimental session. However, at the end of the experiment, participants received the additional $25 \mathrm{CHF}$ based on their subjective achievements.

\section{Questionnaires}

During the first night, participants filled out a general questionnaire for health and personal information (for example related to Body Mass Index, handedness, sex, experience in a sleep laboratory, or sleep habits) where the Edinburgh Inventory for Handedness, ${ }^{21}$ the Pittsburgh Sleep Questionnaire Inventory ${ }^{19}$ and a questionnaire for chronotype $^{18}$ were used. Additionally, in each session, participants filled out a general questionnaire with information regarding their sleep the previous night as well as general health, such as consumption of alcohol, caffeine, drugs, or nicotine. Lastly, they filled out a questionnaire relative to their mood. ${ }^{22}$ In the morning, while still lying in bed, participants were asked to rate their subjective sleep quality, ${ }^{23}$ and again, their mood. ${ }^{22}$ After the last night, they performed the Harvard Group Scale of Hypnotic Susceptibility (high suggestibility $\geq 7$ ). ${ }^{24}$

\section{Memory Measurement}

Episodic memory was tested with a paired-associated learning (PAL) task. ${ }^{20}$ Participants learned a list of 40 semantically related word pairs. Each trial started with the first word of a pair which was presented for $3000 \mathrm{~ms}$, followed by a $500 \mathrm{~ms}$ blank interval which separated the single trials. The words were presented in black font on a white screen via E-Prime (Psychology Software Tools, Pittsburgh). Each pair was presented only once while the order was kept constant. Immediately after learning, participants were confronted with a cued recall test. Here, they had to come up with the corresponding word when the first word was displayed. During the recall test, the word pairs were presented for an infinite amount of time or until the participant pressed enter, then the correct answer was presented straight away for $1000 \mathrm{~ms}$ followed by a $500 \mathrm{~ms}$ blank interval which, again, separated the single trials. A second recall test followed. In this second recall test, the participants did not receive any correction or confirmation of the correct word pair; right after filling in the answer, a 500ms blank interval followed. During recall, the order of the word pairs differed from the learning phase but was kept constant across subjects. A third recall phase took place in the morning. Performance was measured as the percentage of words recalled in the morning relative to the amount of words remembered immediately after learning. Response time was not restricted.

\section{Psychomotor Vigilance Test (PVT)}

In the morning participants performed a psychomotor vigilance test (PVT) which is designed to measure the effect of sleepiness on vigilance. ${ }^{25}$ Subjects were asked to press the space key with their non-dominant hand as soon as they recognized the millisecond counter on the screen, which appeared at random intervals. After the keypress, the reaction time in milliseconds was shown for $1 \mathrm{~s}$.

\section{Polysomnographic Recordings}

To measure sleep, EEG, EMG, EOG and ECG were used. EEG was recorded using 10 single gold-cap-electrodes following the 10-20-EEG-system with a sampling rate of $500 \mathrm{~Hz}$. Impedances were kept below $5 \mathrm{k} \Omega$. Following the AASM guidelines, electrodes were referenced against $\mathrm{Cz}$ during recording and then re-referenced to the mastoids when doing offline analyses. Data were preprocessed with Brain Vision Analyzer 2.0 (Brain Products GmbH, Gilching), filtering the data by $0.3-35 \mathrm{~Hz}$ following the guidelines suggested by the American Association of Sleep. ${ }^{26}$ Two independent sleep scorers visually scored sleep in $30 \mathrm{~s}$ periods based on derivations F4, C4, O4, EOG (left and right), and EMG. Stages N1-N3, REM sleep, and WASO were scored following the AASMguidelines. ${ }^{26}$ Additionally, data were analyzed with an 
automated sleep scoring algorithm (The SIESTA Group Schlafanalyse $\mathrm{GmbH}$, Vienna). These analyses provide more insight on microstructural parameters such as arousals and stage shifts.

\section{Analysis of the EEG Data}

For sleep analysis, we used the SleepTrip toolbox ${ }^{27}$ for Matlab (Mathworks, Natick). Data were preprocessed with Brain Vision Analyzer 2.0 (Brain Products $\mathrm{GmbH}$, Gilching) before spectral analysis. We calculated the average power of oscillatory activity in different frequency bands: slow-wave activity (SWA) $(0.5-4.5 \mathrm{~Hz})$, theta activity $(4.5-8 \mathrm{~Hz})$, alpha activity $(8-11 \mathrm{~Hz})$, slow spindles $(11-13 \mathrm{~Hz})$, fast spindles $(13-15 \mathrm{~Hz})$ and beta activity $(15-30 \mathrm{~Hz})$. In addition, we calculated the ratio between SWA and beta activity as this measure is associated with objective sleep quality. ${ }^{28-30}$ Data from lights off in the evening to lights on in the morning were analyzed, segmented for NREM sleep (N2 and N3 sleep) and REM sleep.

\section{Analysis of Sleep Cycles}

A cycle analysis was performed using Matlab (Mathworks, Natick). The first cycle started with sleep onset. A cycle ends when a REM episode is not followed by another REM episode for 15 mins. For each cycle, we calculated the amount of N1, N2, N3, REM, and WASO as well as the duration of each cycle.

\section{Analysis of ECG}

ECG (electrocardiogram) analysis was carried out using Kubios HRV Premium 3.2.0 (Kubios Oy, Kuopio). Therefore, the ECG signal for pre-sleep (lights off to the first stage of N1) and the whole night (lights off to lights on) was exported in EDF+-format using BrainVisionAnalyzer Version 2.0 (Brain Products GmbH, Gilching). Kubios HRV Premium offers an automatic artifact correction based on the RR series (the interval between two $\mathrm{R}$-signals) to eliminate ectopic beats and artifacts in unfiltered data. ${ }^{31}$ Afterwards, data were analyzed in a time and frequency domain and subsequently used for the calculation of the mean heart rate (mean HR measured in beats per minute) which served as an index for physiological arousal. ${ }^{32}$ Furthermore, Kubios provides an index to analyze the tone of the parasympathetic nervous system (PNS) and sympathetic nervous system (SNS). We used the PNSand SNS-indexes to evaluate an appropriate measure for physiological stress. The PNS-index is a PNS tone index based on mean RR whereas the SNS-index is an SNS tone index to evaluate stress which is based on Mean HR. ${ }^{31}$

\section{Statistical Analysis}

According to our experimental manipulation before sleep (see section "Instruction"), our main outcome variables were subjective and objective sleep quality, SOL, WASO, NWAK and sleep depth. For each of these main outcome variables, a separate repeated-measures analysis of variance (ANOVA) with the within-subject factor condition ("bad", "good", and "neutral") was calculated. In a separate analysis, we used a 2x3 Analysis of Variance with the within-subject factors "type of parameter" (subjective vs objective) and condition ("bad", "good", and "neutral") for the five main outcome variables. In addition, we report Pearson's correlation coefficient (r) for the strength of association between these objective and subjective sleep parameters.

In exploratory analyses we analyzed further sleep parameters, vigilance, sleep-associated memory consolidation, and heart rate variability (HRV) using an ANOVA for repeated measures with the within-subject factor condition ("bad", "good", "neutral"). For oscillatory power during NREM and REM sleep, we used a $3 \times 2 \times 3$ ANOVA for repeated measurements using the factors "condition" (bad, good, neutral), "hemisphere" (left, right) and "topography" (frontal, central, parietal). And we report Pearson's correlation coefficient ( $r$ ) for the strength of association between sleep parameters and vigilance.

Post-hoc paired t-tests with a Bonferroni correction were conducted in case of significant main effects or interaction effects. We set the level of significance to $p \leq 0.05$ and reported effect sizes $\left(\eta^{2}\right)$ only for significant data. Data were analyzed using IBM SPSS Statistics 25 (IBM Corp., Armonk) and R Studio (RStudio Team, Boston). Results are presented as means \pm standard errors of the mean (SEM).

\section{Data Availability}

Datasets analyzed during the current study are available online on https://osf.io/asz94/.

\section{Results \\ Subjective Sleep Parameters}

According to the instructions, our primary outcome variables were subjective sleep quality, subjective SOL, subjective WASO, subjective NWAK, and subjective sleep depth. All subjective variables were measured with the SFA-R questionnaire. ${ }^{23}$ 
In accordance with our hypothesis, participants were able to subjectively worsen, but not to improve their sleep. In the "bad" sleep condition, participants rated their sleep quality in the morning as worse $(16.23 \pm 0.98$, scale from 7 to 35 , higher values indicating better sleep quality) compared to the "good" $(25.05 \pm 1.20)$, and "neutral" conditions $(24.55 \pm 1.11)$ (repeated-measures ANOVA with the factor condition ("bad", "good", "neutral"), F(2, $20)=24.55, p<0.001, \eta^{2}=0.71$, see Figure 2A, Table 1). Post-hoc pairwise comparisons confirmed that with the instruction to sleep "bad" participants rated their sleep quality significantly worse as compared to the "neutral" and "good" conditions (both $p<0.001$ ). "Good" and "neutral" conditions did not differ significantly $(p>0.50)$.

We observed a similar pattern for SOL, WASO, NWAK and sleep depth (see Figure 2B-E, for post hoc pairwise comparisons).

\section{Objective Sleep Parameters}

As was reported for subjective sleep parameters, on the objective level participants were able to worsen but not to improve their sleep by simply wanting to do so. Similar to the subjective ratings, our primary outcome variables for objective sleep parameters were sleep efficiency (taken as objective measure of sleep quality), SOL, WASO, NWAK and amount of SWS in minutes (taken as objective measure of sleep depth).

As predicted and in line with the subjective measures of sleep quality, participants who were instructed to sleep "bad" showed a reduced sleep efficiency with $93.67 \pm$ $0.97 \%$ compared to $97.45 \pm 0.57 \%$ in the "neutral" condition and $96.91 \pm 0.66 \%$ in the "good" condition $(F(2,42)=$ $\left.12.69, \mathrm{p}<0.001, \eta^{2}=0.377\right)$. Post-hoc pairwise comparisons confirmed that there was a significant reduction of sleep efficiency in the "bad" condition compared to both the "neutral" $(p<0.001)$ and "good" $(p=0.004)$ conditions. The latter two conditions did not differ ( $p>0.20$, see Figure $2 \mathrm{~F}$ and Table 1).

We observed the same pattern for objective SOL, as people took roughly 15 mins longer to fall asleep when they were asked to sleep "bad" (see Figure $2 \mathrm{G}$ and Table 1). In addition, they increased their NWAK: in the "bad" sleep condition participants awoke $8.05 \pm 0.87$ times, whereas they woke up only $4.73 \pm 0.65$ times in the "neutral" condition and $5.68 \pm$
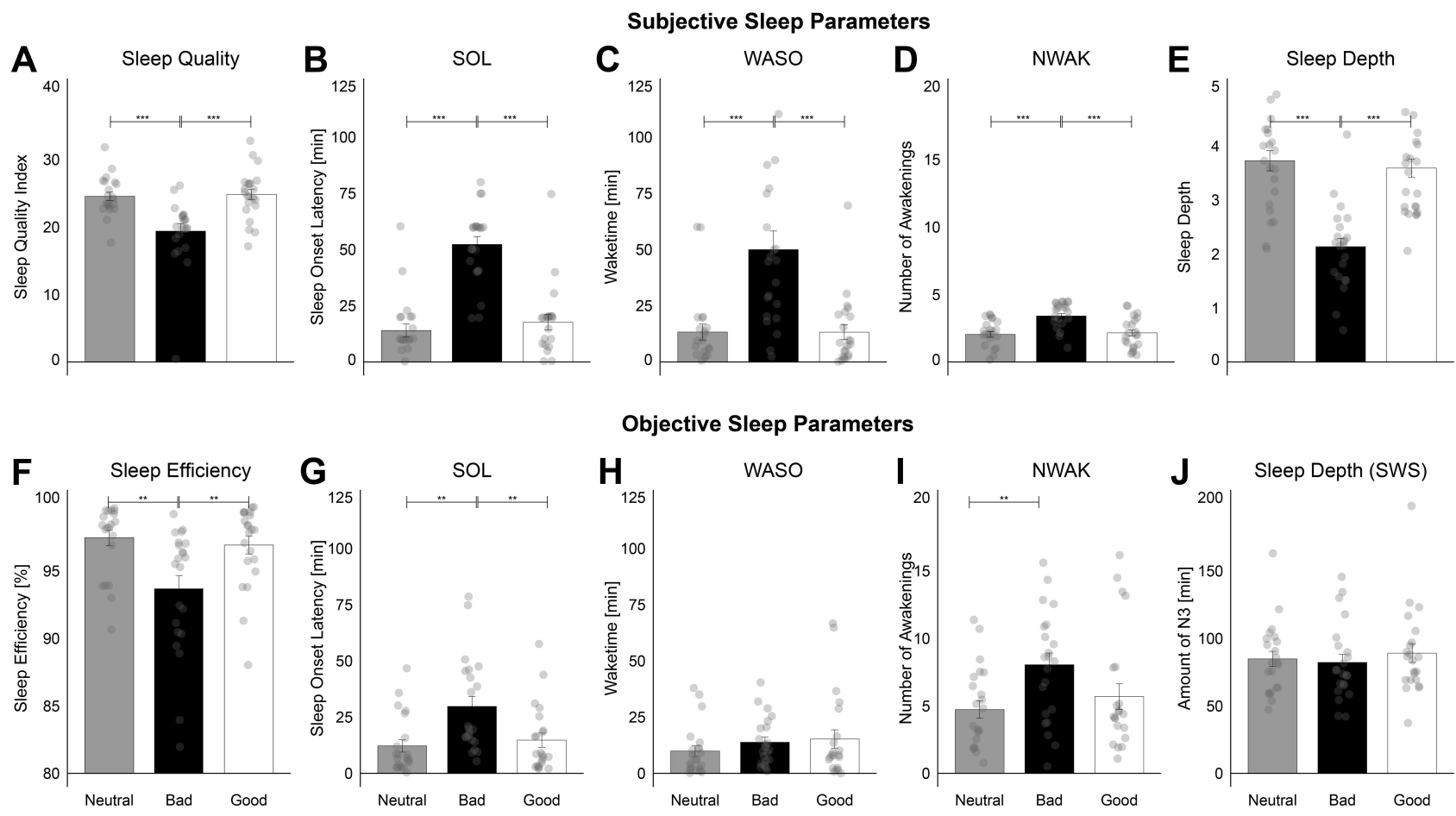

Figure 2 Effects of instructions on subjective and objective sleep parameters. On the subjective level, after being instructed to sleep "bad" (black bars), participants reported (A) to sleep worse (sleep quality), (B) took more time to fall asleep (sleep onset latency (SOL)), (C) spent more time awake after sleep onset (WASO), (D) woke up more often (NWAK) and (E) slept less deep (sleep depth) as compared to the night with the instruction to sleep good (white bars) and as compared to the night without specific instructions ("neutral", grey bars). The instruction to sleep "good" (white bars) did not alter subjective evaluations of sleep. For objective sleep parameters, the instruction to sleep "bad" (F) reduced sleep efficiency as an objective measurement for sleep quality, (G) extended SOL and (I) increased the NWAK during the night as compared to the night without instructions ("neutral"). No effect was observed for WASO $(\mathbf{H})$ and the time spent in SWS (J). Again, no effect occurred after the instruction to sleep "good". Means \pm standard errors of the mean are indicated. Significant pair-wise comparisons from post hoc tests are indicated by $* * p \leq 0.0 \mathrm{I}$. ***p $\leq 0.00 \mathrm{I}$. 
Table I Sleep Parameters for the Three Conditions (Subjective and Objective)

\begin{tabular}{|c|c|c|c|c|}
\hline & \multirow{2}{*}{$\begin{array}{l}\text { Bad } \\
M \pm \text { SEM }\end{array}$} & \multirow{2}{*}{$\begin{array}{l}\text { Good } \\
M \pm \text { SEM }\end{array}$} & \multirow{2}{*}{$\frac{\text { Neutral }}{M \pm \text { SEM }}$} & \multirow[t]{2}{*}{ F-Test } \\
\hline & & & & \\
\hline \multicolumn{5}{|l|}{ Subjective Sleep Parameters } \\
\hline Sleep Quality & $20.28 \pm 0.592$ & $24.833 \pm 0.77$ & $24.547 \pm 0.61$ & $24.253 * * *$ \\
\hline $\mathrm{SOL}[\mathrm{min}]$ & $52.27 \pm 3.51$ & $17.64 \pm 3.44$ & $14.02 \pm 2.97$ & $53.988 * * *$ \\
\hline WASO [min] & $39.55 \pm 8.04$ & $9.09 \pm 2.04$ & $10.55 \pm 3.66$ & $11.273^{* * *}$ \\
\hline NWAK & $3.41 \pm 0.18$ & $2.16 \pm 0.23$ & $2.05 \pm 0.21$ & $20.573 * * *$ \\
\hline Sleep Depth & $2.14 \pm 0.15$ & $3.59 \pm 0.17$ & $3.73 \pm 0.19$ & $47.513 * * *$ \\
\hline \multicolumn{5}{|l|}{ Objective Sleep Parameters } \\
\hline Sleep Efficiency [\%] & $93.67 \pm 0.97$ & $96.91 \pm 0.66$ & $97.45 \pm 0.57$ & $12.09 * * *$ \\
\hline SOL [min] & $29.75 \pm 4.46$ & $14.77 \pm 3.14$ & $12.21 \pm 2.75$ & $12.864 * * *$ \\
\hline WASO [min] & $13.84 \pm 2.28$ & $15.25 \pm 4.12$ & $9.89 \pm 2.341$ & 1.126 \\
\hline NWAK & $8.05 \pm 0.87$ & $5.68 \pm 0.95$ & $4.73 \pm 0.65$ & $6.98 * *$ \\
\hline NWAK + Movements & $16.46 \pm 1.60$ & $10.68 \pm 1.20$ & $1 \mathrm{I} .4 \mathrm{I} \pm 1.1 \mathrm{I}$ & $10.34 * * *$ \\
\hline Duration per Awakening [min] & $1.63 \pm 0.38$ & $1.59 \pm 0.47$ & $1.23 \pm 0.30$ & 0.40 \\
\hline $\mathrm{NI}$ [min] & $34.89 \pm 3.96$ & $39.52 \pm 3.15$ & $33.61 \pm 2.53$ & 1.68 \\
\hline $\mathrm{N} 2$ [min] & $211.73 \pm 6.20$ & $231.61 \pm 7.32$ & $234.80 \pm 6.49$ & $4.57^{*}$ \\
\hline N3 [min] & $82.18 \pm 6.05$ & $89.09 \pm 6.89$ & $84.80 \pm 5.61$ & 1.05 \\
\hline REM [min] & $97.89 \pm 4.28$ & $88.30 \pm 4.70$ & $100.02 \pm 4.25$ & 3.05 \\
\hline Move [min] & $3.16 \pm 0.81$ & $2.21 \pm 0.61$ & $2.55 \pm 0.6 I$ & 1.60 \\
\hline NI [\%] & $7.83 \pm 0.87$ & $8.47 \pm 0.67$ & $7.20 \pm 0.53$ & 2.02 \\
\hline N2 [\%] & $47.71 \pm 1.14$ & $49.69 \pm 1.53$ & $50.37 \pm 1.35$ & 1.46 \\
\hline N3 [\%] & $18.46 \pm 1.29$ & $19.11 \pm 1.50$ & $18.24 \pm 1.25$ & 0.35 \\
\hline REM [\%] & $22.11 \pm 0.92$ & $18.92 \pm 0.97$ & $21.43 \pm 0.88$ & $4.77^{*}$ \\
\hline Move [\%] & $0.71 \pm 0.18$ & $0.46 \pm 0.13$ & $0.54 \pm 0.13$ & 2.28 \\
\hline WASO [\%] & $3.16 \pm 0.54$ & $3.35 \pm 0.92$ & $2.13 \pm 0.51$ & 2.55 \\
\hline TST [min] & $443.68 \pm 97.86$ & $465.98 \pm 101.70$ & $466.00 \pm 102.66$ & $8.98^{* * * *}$ \\
\hline SWS Latency [min] & $16.77 \pm 5.97$ & $17.00 \pm 4.80$ & $17.00 \pm 5.33$ & 0.01 \\
\hline REM Latency [min] & $78.34 \pm 1.71$ & $97.64 \pm 33.37$ & $82.93 \pm 2.03$ & 2.14 \\
\hline \multicolumn{5}{|l|}{ Memory } \\
\hline Encoding (evening) & $32.77 \pm 1.06$ & $32.86 \pm 1.05$ & $31.18 \pm 0.88$ & 2.362 \\
\hline Recall (morning) & $31.86 \pm 1.08$ & $31.64 \pm 1.21$ & $30.05 \pm 0.97$ & 2.698 \\
\hline Consolidation [\%] & $97.22 \pm 0.97$ & $95.98 \pm 1.34$ & $96.40 \pm 1.53$ & 0.508 \\
\hline \multicolumn{5}{|l|}{ Vigilance (Morning) } \\
\hline Reaction Time (RT) & $368.12 \pm 12.14$ & $332.42 \pm 7.52$ & $342.48 \pm 7.79$ & $7.994 * * *$ \\
\hline Reactions & $77 \pm 0.55$ & $76.27 \pm 0.66$ & $77.36 \pm 0.58$ & 0.834 \\
\hline Errors & $1.5 \pm 8.92$ & $12 \pm 0.38$ & $1.27 \pm 0.40$ & 1.409 \\
\hline
\end{tabular}

Notes: Subjective parameters are based on subjective ratings in the SF-A-R ${ }^{23}$. Objective values are based on polysomnographic recordings. Non-rapid eye movement (NREM)-sleep, stage I, 2, and 3 sleep (NI, N2, N3), rapid eye movement sleep (REM), waketime after sleep onset (WASO), total sleep time (TST), sleep onset latency (SOL), slow-wave sleep latency (SWS latency), REM sleep latency (REM latency) are all measured in minutes [min] and the percentages indicate parietal percentage of TST [\%]. For memory, numbers indicate absolute or relative values of correctly recalled words that were presented in the evening (learning phase with first recall) and in the morning (retrieval phase with second recall). Consolidation refers to the difference in performance between learning and retrieval phases. For vigilance, the reaction time (RT), the number of reactions, and amount of errors during the 10 minutes of the psychomotor vigilance task (PVT) were measured. Values are means \pm standard error of mean (SEM). * Indicates $\mathrm{p} \leq 0.05$ and $* *$ indicates $\mathrm{p} \leq 0.0 \mathrm{I} * * *$ indicates $\mathrm{p} \leq 0.00 \mathrm{I}$

0.95 times in the "good" condition $(\mathrm{F}(2,42)=6.98, \mathrm{p}=0.002$, $\eta^{2}=0.249$ (see Figure 2I). This difference was even more pronounced when movements were included in the number of awakenings $\left(F(2,42)=10.34, p<0.001, \eta^{2}=0.330\right.$, see Table 1).
In contrast to our expectations, instructions were not able to influence the objective time spent awake (WASO, F $(2,42)=1.03, \mathrm{p}>0.30$, see Figure $2 \mathrm{H}$, Table 1$)$ or the objective depth of sleep (sleep stage N3 F(2,42) $=1.05$, $\mathrm{p}=0.358$, see Figure 2J, Table 1). 
Table 2 Arousals and Stage Shifts

\begin{tabular}{|l|l|l|l|l|}
\hline & Neutral & Bad & Good \\
\cline { 2 - 4 } & M \pm SEM & M \pm SEM & M \pm SEM \\
\hline Arousal & & & \\
Arousals in TST & $159.10 \pm 28.37$ & $159.19 \pm 25.10$ & $167.95 \pm 28.80$ \\
Arousals in NREM & $141.57 \pm 24.79$ & $139.48 \pm 23.24$ & $153.10 \pm 29.69$ \\
Arousals in REM & $17.52 \pm 7.42$ & $16.71 \pm 5.46$ & $14.86 \pm 3.71$ & 1.81 \\
Arousal Index (TST) & $20.86 \pm 3.65$ & $21.86 \pm 3.51$ & $22.17 \pm 3.66$ \\
Arousal Index NREM & $24.07 \pm 3.63$ & $25.21 \pm 3.72$ & $25.37 \pm 3.97$ & 1.67 \\
Arousal Index REM & $10.4 \pm 3.69$ & $10.51 \pm 2.92$ & $9.90 \pm 2.41$ \\
\hline Stage shifts from any sleep stage to & & & 1.18 \\
Wake & $10.10 \pm 2.84$ & $13.76 \pm 2.18$ & $10.90 \pm 1.96$ \\
NI & $42.52 \pm 7.64$ & $43.86 \pm 7.42$ & $45.71 \pm 9.17$ \\
N2 & $55.48 \pm 10.91$ & $54.86 \pm 9.60$ & $57.38 \pm 13.09$ \\
N3 & $19.67 \pm 6.11$ & $18.10 \pm 2.84$ & $18.76 \pm 5.67$ \\
REM & $7.76 \pm 1.31$ & $7.86 \pm 1.53$ & $6.71 \pm 0.87$ \\
\hline
\end{tabular}

Notes: Analysis with an automatic sleep scoring algorithm (The SIESTA Group Schlafanalyse GmbH, Vienna) providing the number of arousals, an arousal index and stage shifts. Number of arousals as well as the arousal index were analyzed for total sleep time (TST), non-rapid eye movement as well as rapid eye movement sleep (REM). Further stage shifts from any sleep stage to wake and to NREM-sleep, stage I, 2, and 3 sleep (NI, N2, N3) are shown. Values are means \pm standard error of mean (SEM). ** Indicates $\mathrm{p} \leq 0.01$

The exploratory analysis of total sleep time also showed a reduced sleep time in the "bad" condition $(\mathrm{F}(2,42)=8.98$, $\mathrm{p}=0.001, \eta^{2}=0.30$, see Table 1$)$. Note that differences in SOL directly affect total sleep time in our study, as all participants were awakened after exactly $8 \mathrm{hrs}$ of time in bed. After correcting for differences in SOL, total sleep time did not differ between conditions $(\mathrm{F}(2,42)=1.39, \mathrm{p}>0.20)$. Explorative analysis on other sleep parameters did not reveal any significant differences for N1, movement time, SWS latency and REM latency sleep (all $p>0.10$ ). In contrast, time spent in stage N2 sleep was significantly shorter in the "bad" condition ( $211.73 \pm 6.20$ mins $)$ as compared to the "neutral" (234.80 \pm 6.49 mins) and "good" $(231.61 \pm 7.32$ mins, $\left.F(2,42)=4.57, p=0.016, \eta^{2}=0.18\right)$ conditions. However, this effect was, again, fully explained by an increase in SOL of 20 min the "bad" condition ( $p<0.60$, after correction for SOL). Interestingly, REM sleep appeared to be shorter in the "good" condition $(88.30 \pm 4.70 \mathrm{mins})$ as compared to "neutral" (100.02 \pm 4.25 mins $)$ and "bad" conditions (97.89 \pm 4.28 mins). The effect only reached a statistical trend $(p=0.058)$, but was statistically significant when considering the percentage of REM sleep relative to total sleep length $\left(\mathrm{F}(2,42)=4.77, \mathrm{p}=0.014, \eta^{2}=0.19\right)$. This effect cannot be explained by SOL as the effect was still present after correcting for differences in SOL between the conditions $\left(\mathrm{F}(2.42)=7.63, \mathrm{p}=0.001, \eta^{2}=0.27\right.$; see Table 1 for an overview).
We also considered microstructural parameters such an arousal index and the amount of stage shifts between the different sleep stages. Results of the arousal index analysis are shown in Table 2. Additionally, we analyzed the stage shifts between different sleep stages and showed a significant effect of the condition on the shifts from any sleep stage to wake $\left(\mathrm{F}(2,40)=5.24, \mathrm{p}=0.010, \eta^{2}=\right.$ $0.21)$ but not between the sleep stages. Post hoc comparison confirmed significantly more stage shifts from any sleep stage to wake in the "bad" $(13.76 \pm 2.18)$ compared to the "neutral" $(10.10 \pm 2.84)$ condition. The other conditions did not differ (all $\mathrm{p}>0.27$ ).

We also analyzed whether the type of instruction before sleep influenced the cyclic structure of sleep. The amount of cycles was of $4.36 \pm 0.12$ over all participants and in each condition ("bad": $4.36 \pm 0.19$; "good": $4.36 \pm$ 0.23 ; "neutral": $4.36 \pm 0.19$ ). It did not differ for the three conditions $(F(2,20)=0, p=1.00)$. Also, the mean duration of the first four cycles was not affected by the different instructions before sleep (for further information see sup plementary material Table S1).

\section{Effect of Pre-Sleep Instructions on Oscillatory Power During NREM and REM Sleep}

In addition to sleep architecture, we also analyzed the effects of intentions on oscillatory power in different frequency bands (slow-wave activity (SWA), theta, alpha, slow spindle, fast spindle and beta band). We observed no significant 
main effects of the factor condition (bad, good, neutral) on oscillatory power during NREM sleep (all $\mathrm{p}>0.10$ ). Furthermore, no significant interaction was found for the factor "condition" with the factors "hemisphere" (left, right), "topography" (frontal, central, parietal), or both (all $p \geq 0.085$ ). Moreover, regarding the ratio between power in the SWA band and beta band, which is considered an indicator of objective sleep quality, no effects nor interactions with the factor condition were observed ( $p \geq 0.085$ ). Also, during REM sleep we did not find any main effects or interactions with the factor condition $(p>0.27$, for an overview see supplementary material Table S2).

\section{Comparing Objective and Subjective Sleep Parameters}

Participants generally overestimated the time it took them to fall asleep as well as the time they spent awake during the night. The degree of overestimation was largest in the "bad" condition: participants reported an SOL of $52.27 \pm 3.51 \mathrm{~min}$, whereas they actually fell asleep after $29.75 \pm 4.46 \mathrm{~min}$, resulting in an overestimation of ca. $23 \mathrm{~min}$. In contrast, they only overestimated SOL by 5 and 2 min in the "good" and "neutral" conditions, respectively (main effect subjective vs objective SOL: $F(20,1)=54.08, p<0.001, \eta^{2}=0.73$; interaction with condition: $F(19,2)=11.62, p=0.001, \eta^{2}$ $=0.55)$. Similarly, in the "bad" condition participants reported having spent $39.55 \pm 8.04$ min WASO, whereas the actual time spent awake was of $13.84 \pm 6.18$ mins (overestimation of ca. $26 \mathrm{~min}$ ). In contrast, overestimation of WASO was only $5 \mathrm{~min}$ in the "good" condition and less than $1 \mathrm{~min}$ in the "neutral" condition (main effect subject vs objective WASO: $F(20,2)=7.75, p=0.003, \eta^{2}$ $=0.44$; interaction with condition, $\mathrm{F}(20,2)=4.83, \mathrm{p}=0.019$, $\eta^{2}=0.33$ ). Thus, the degree of overestimation was larger in the "bad" condition as compared to the "good" and "neutral" conditions (see Table 1).

In spite of the high overestimation of SOL in the "bad" condition, subjective estimates of SOL were still positively and significantly correlated with the objective measure of sleep onset (Pearson's $r=0.58, \mathrm{p}=0.004$; see Table 3). Also, in the "good" condition, subjective and objective SOL were moderately to highly correlated ( $r=0.64, p=0.001)$. Only in the "neutral" condition no significant or positive correlation was observed between these two measurements $(r=0.23, \mathrm{p}>0.30)$. WASO was not significantly correlated in any of the three conditions ("bad": $r=0.14, p>0.50$, "good": $r=0.31, p>$ 0.17 , "neutral": $\mathrm{r}=0.27, \mathrm{p}>0.23$ ). In addition, objective NWAK was negatively correlated with subjective sleep quality $(\mathrm{r}=-0.56, \mathrm{p}=0.004)$ and sleep depth $(\mathrm{r}=$ $-0.65, \mathrm{p} \leq 0.001)$ in the "neutral" condition (see Table 3).

Table 3 Correlation Between Objective and Subjective Sleep Parameters

\begin{tabular}{|c|c|c|c|c|c|c|c|}
\hline & & & \multicolumn{5}{|c|}{ Objective Sleep Parameters } \\
\hline & & & SE & SOL & WASO & NWAK & Depth (N3) \\
\hline \multirow{15}{*}{ Subjective Sleep Parameters } & Bad & Sleep Quality & -0.059 & 0.067 & -0.203 & -0.108 & 0.05 \\
\hline & & SOL & $-0.58 * *$ & $0.58 * *$ & 0.19 & -0.09 & 0.183 \\
\hline & & WASO & 0.115 & -0.13 & 0.142 & 0 & 0.099 \\
\hline & & NWAK & 0.376 & -0.36 & 0.222 & 0.24 & -0.256 \\
\hline & & Depth & 0.02 & -0.015 & -0.274 & -0.35 & 0.24 \\
\hline & Good & Sleep Quality & 0.192 & -0.189 & -0.183 & -0.35 & -0.051 \\
\hline & & SOL & $-0.64 * *$ & $0.64 * *$ & 0.07 & 0.22 & -0.284 \\
\hline & & WASO & -0.162 & 0.16 & 0.305 & 0.079 & 0.028 \\
\hline & & NWAK & -0.283 & 0.28 & 0.294 & 0.197 & 0.066 \\
\hline & & Depth & 0.23 & -0.229 & 0.039 & -0.413 & 0.124 \\
\hline & Neutral & Sleep Quality & 0.221 & -0.221 & -0.257 & $-0.555^{* *}$ & 0.209 \\
\hline & & SOL & -0.233 & 0.232 & 0.1 & 0.357 & 0.04 \\
\hline & & WASO & -0.181 & 0.176 & 0.266 & 0.221 & -0.101 \\
\hline & & NWAK & -0.26 & 0.258 & $0.485^{*}$ & 0.256 & -0.242 \\
\hline & & Depth & $0.34 I$ & -0.34 & -0.109 & $-0.653^{* *}$ & 0.094 \\
\hline
\end{tabular}

Notes: Correlations were calculated separately for "bad", "good” and "neutral” conditions for subjective (measured by SF-A-R ${ }^{23}$ : Sleep quality, sleep onset latency (SOL), wake time after sleep onset (WASO), numbers of awakenings (NWAK) and sleep depth) and objective sleep parameters (Sleep quality = sleep efficiency (SE), SOL, WASO, NWAK, depth $=$ sleep stage N3 in minutes). * Indicates $p \leq 0.05$ and $* *$ Indicates $p \leq 0.01$ 


\section{Memory Consolidation and Vigilance}

In our study, we also tested whether the instructions to sleep "good", "neutral" or "bad" could affect memory consolidation during sleep and vigilance the next morning. Pre-sleep instructions did not affect overnight memory formation differentially $(F(2,18)=0.508, p>0.60)$. However, the pre-sleep instruction to sleep "bad" resulted in slower reaction times in the Psychomotor Vigilance Test (PVT) directly after waking up. Two participants were excluded because of the high amount of errors $(>3$ SD). Vigilance (as indicated by reaction time) was significantly lower after sleep in the "bad" condition (368.12 $\pm 12.14 \mathrm{~ms})$ as compared to the "neutral" (342.48 \pm 7.79 $\mathrm{ms})$ and "good" conditions $(332.42 \pm 7.52 \mathrm{~ms}, \mathrm{~F}(2,42)=$ $7.99 ; p=0.001, \eta^{2}=0.25$ see Figure 3 , for post hoc tests). In an exploratory analysis, we examined whether the decreased vigilance after the "bad" night, as compared to the "neutral" night, could be explained by differences in subjective or objective sleep parameters.
Thus, we correlated the difference in reaction time on the PVT task between "bad" and "neutral" conditions, with the difference in sleep parameters between the "bad" and "neutral" conditions. Interestingly, neither any objective nor any subjective sleep parameters correlated with the decrease in reaction time in the "bad" condition (corrected for "neutral") (all p >0.10; see Table 4). When calculating the difference between "good" and "neutral" conditions, we observed several correlations with subjective but not objective sleep parameters: the difference in reaction time correlated with differences in subjective ratings of sleep quality $(r=-0.627, p=0.002)$ and with the feeling after waking-up $(r=-0.572, p=$ $0.005)$ between the two conditions. As positive differences in sleep quality ratings (eg, better sleep quality in "good" vs "neutral" conditions) are predictive for a more negative difference in reaction times (eg, fast reaction time in "good" vs "neutral" conditions), negative correlations are to be expected.
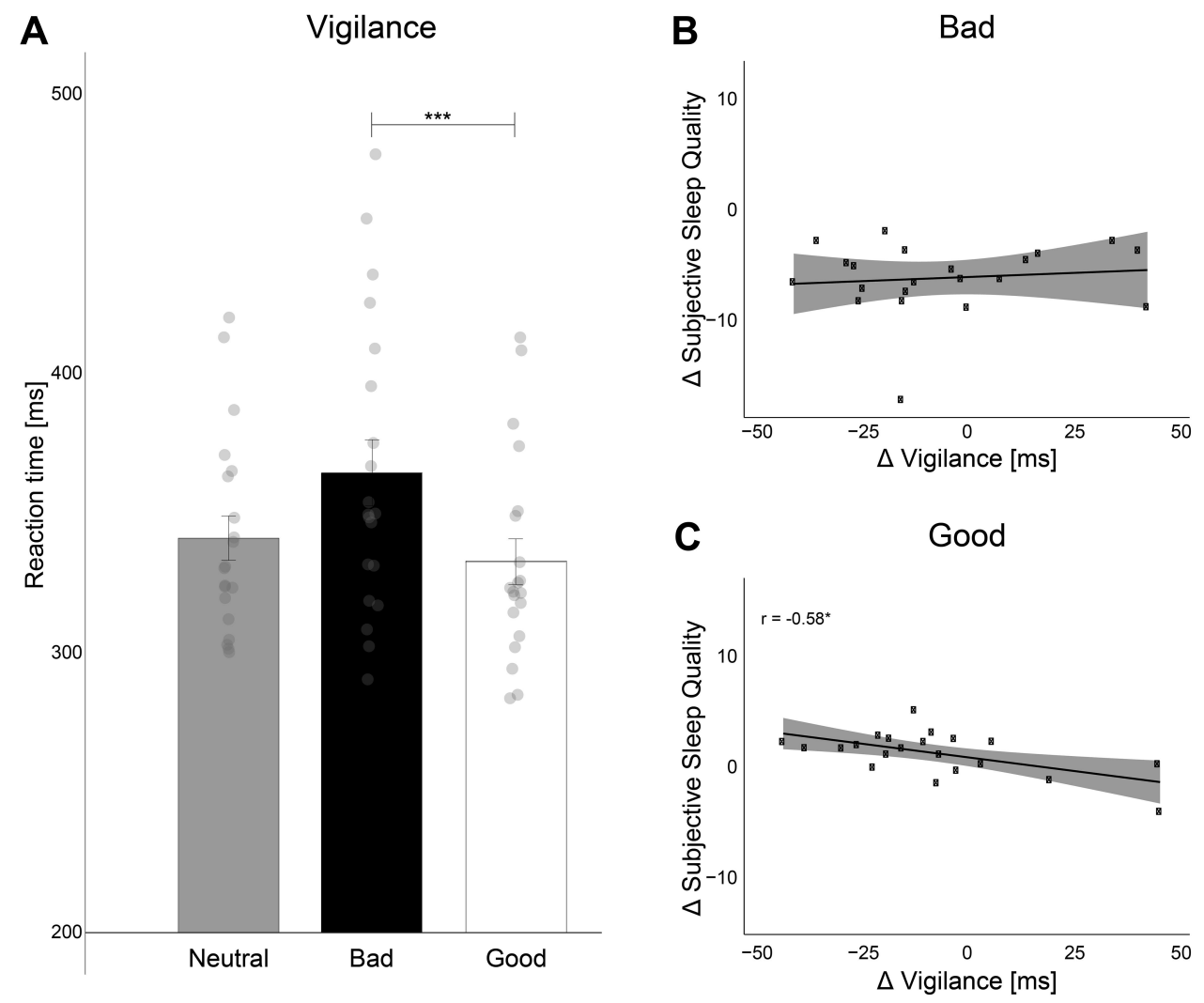

Figure 3 Effects of the instructions to change sleep quality on morning vigilance. (A) The instruction to sleep "bad" increased reaction in the psychomotor vigilance test (PVT) as compared to the "good" and "neutral" night (main effect condition $p=0.00 \mathrm{l}$ ). Post-hoc tests revealed a significant difference between "bad" and "good" instructions ( $p=0.034)$ but not between "bad" and "neutral" ( $p=0.11)$ or "good" and "neutral" $(p>0.46)$. Means \pm standard errors of the mean are indicated. (B) The overall increase in reaction time in the PVT in the "bad" condition (corrected for performance in the "neutral" condition) was not correlated with any of our main subjective or objective sleep parameters (see Table 3). (C) In the "good" condition, the change in reaction (corrected for the neutral condition) correlated negatively with the change in subjective sleep between good and neutral sleep conditions. The direction of the correlation were expected, as better sleep correlated with faster reaction times and better vigilance. $*$ indicates $\mathrm{p} \leq 0.05$. *** Indicates $\mathrm{p} \leq 0.00 \mathrm{I}$. 
Table 4 Correlation of Vigilance with Subjective and Objective Sleep Parameters

\begin{tabular}{|l|l|l|l|l|}
\hline \multirow{2}{*}{ Vigilance } & $\Delta$ Bad & $\Delta$ Good & $\Delta$ Bad & $\Delta$ Good \\
\cline { 2 - 5 } & \multicolumn{2}{|l|}{ Subjective Sleep } & \multicolumn{2}{l|}{ Objective Sleep } \\
\hline$\Delta$ Sleep Quality & 0.092 & $-0.627^{* * *}$ & 0.030 & -0.008 \\
$\Delta$ SOL & -0.047 & -0.289 & 0.009 & 0.009 \\
$\Delta$ WASO & -0.065 & 0.133 & -0.034 & 0.309 \\
$\Delta$ NWAK & 0.081 & 0.169 & -0.044 & $0.413^{* *}$ \\
$\Delta$ Sleep Depth & -0.058 & -0.194 & 0.311 & 0.201 \\
\hline
\end{tabular}

Notes: Correlations were calculated separately for the "good" and "bad" conditions. All parameters are corrected for the values obtained in the neutral condition ( $\Delta$ Bad: "bad" - "neutral" and $\Delta$ Good: "good" - "neutral", respectively). ** Indicates $p \leq 0.01$. ****Indicates $p \leq 0.001$.

Table 5 Correlation of Heart Rate with Subjective and Objective Sleep Parameters

\begin{tabular}{|l|l|l|l|l|}
\hline Heart Rate [bpm] & $\Delta$ Bad & $\Delta$ Good & $\Delta$ Bad & $\Delta$ Good \\
\cline { 2 - 5 } & \multicolumn{2}{|l|}{ Subjective Sleep } & \multicolumn{2}{|c|}{ Objective Sleep } \\
\hline$\Delta$ Sleep Quality & 0.193 & 0.013 & 0.012 & 0.283 \\
$\Delta$ SOL & 0.029 & -0.262 & 0.011 & -0.278 \\
$\Delta$ WASO & -0.112 & -0.304 & 0.245 & $0.453^{*}$ \\
$\Delta$ NWAK & -0.017 & 0.154 & 0.085 & 0.244 \\
$\Delta$ Sleep Depth & 0.085 & 0.029 & -0.037 & 0.313 \\
\hline
\end{tabular}

Notes: Correlations were calculated separately for the "good" and "bad" conditions. All parameters are corrected for the values obtained in the neutral condition ( $\Delta$ Bad: "bad" - "neutral" and $\Delta$ Good: "good" - "neutral"). * Indicates $p \leq 0.05$.

\section{Heart Rate and Heart Rate Variability Before Sleep}

As the instruction to sleep "bad" had a clear negative impact on subjective and objective sleep parameters, we examined whether the difference in physiological arousal before falling asleep could explain these changes. First, we analyzed average heart rate before falling asleep (from lights off to the first sign of sleep (N1)). Surprisingly, participants had the lowest heart rate before sleep in the "bad" condition (65.99 bpm \pm 1.05 ) as compared to the other conditions ("good" $=69.73 \pm 1.34$; "neutral" $=67.16 \pm 1.21$ ). This decrease in heart rate reached a statistical trend $(\mathrm{F}(2,39)=$ $\left.2.52, p=0.097, \eta^{2}=0.03\right)$. Regarding heart rate variability, neither of the indexes for activity of the sympathetic tone (SNS index) nor for the parasympathetic tone (PNS index) were affected by the pre-sleep instructions (both $\mathrm{p}>0.30$ ). No significant correlations between heart rate/heart rate variability and any subjective or objective sleep parameters were observed (all $p>0.05$ ). However, one exception to this is found in the differences in heart rate between the "good" and "neutral" conditions as they were positively correlated with the difference in objective WASO $(r=0.453, p=$ 0.017 , see Table 5).

\section{Subjective Reports How Participants Manipulated Their Sleep}

Participants reported different methods in order to manipulate their sleep according to the pre-sleep instructions. To subjectively affect their sleep in a negative way, most participants reported trying to keep their eyes open (7 reports), to do exercises with fingers, legs or arms (2 reports) or to lie in an uncomfortable sleep position (4 reports). With regard to mental strategies that were implemented to sleep "bad", participants reported generally activating their thinking (12 reports), thinking about their planning and their to-dolist for the next day/week ( 8 reports) and thinking about negative/sad events (6 reports). One participant mentioned that he got upset about not falling asleep and another one recited a poem.

To affect sleep positively, participants mentioned using relaxation techniques such as breathing techniques, meditation and mind-clearing ("do not think at all"), as well as muscle relaxation (11 times) and finding a comfortable and relaxing position to sleep in. In addition, participants mentioned thinking about positive memories and positive situations six times. Another strategy used by various participants was the active generation of sleep-related thoughts (eg, "fall asleep fast", "sleep as usual" (as they knew they were healthy sleepers) or "fall asleep").

\section{Discussion}

Our results support the previously mentioned prediction that intention can decrease sleep quality but not improve it in healthy young participants. On the subjective level, all of our five primary outcome measures (sleep quality, SOL, WASO, NWAK, and sleep depth) show this pattern of results. On the level of objective sleep parameters, three out of five primary outcome values (SE, SOL, and NWAK) also support this pattern of results, whereas no effects of voluntary sleep manipulation were observed for WASO and the amount of SWS.

As we examined sleep in young healthy participants, their sleep during the night was already very good without having to provide them with any instructions: on average they had a high sleep efficiency (over 97\%), they fell asleep after 12 mins and had only 2\% WASO during the total of 8 hrs of sleep. Average sleep stage duration and sleep stage distribution also were in the normal range. As this sleep 
pattern is close to optimal sleep, there is probably not much room for improvement of sleep simply by wanting to do so. To achieve good sleep, participants reported using breathing or relaxation techniques as well as thinking positive thoughts. Still, these techniques did not produce any further improvement in sleep. However, most of these techniques need a certain degree of training, a reason which may help explain why our physiological parameters of sleep showed no changes. ${ }^{35}$ For example, oscillatory power during the night - and particularly SWA, which is an important measure of the homeostatic component of sleep - was not altered by our experimental manipulation, which might be interpreted as evidence that the sleeping brain is regulated mainly in a physiological manner. ${ }^{33,36}$ However, as sleep was close to optimal, our null finding does not imply that these techniques or intentions in general, are ineffective in improving sleep. We can only conclude that intention cannot further improve very good sleep in healthy young participants. In patients with insomnia, relaxation techniques have been shown to improve some aspects of sleep. ${ }^{37-39}$ In addition, the anticipation of receiving a treatment or a medication thinking that it will help sleep (known as the "placebo" effect) can improve subjective and also some objective sleep variables in persons with disturbed sleep. $^{40-42}$ Also in healthy sleeper, sleep improvements (extension of SWS and increases of SWA) are possible for example, using music, ${ }^{29,43}$ hypnotic suggestions, ${ }^{44,45}$ or rocking bed movements). ${ }^{46}$

Some participants also reported that they generated sleep-related thoughts (eg, "fall asleep fast"), which is in line with a paradoxical negative rebound. ${ }^{12}$ Previous studies have suggested that wanting to fall asleep, prolongs sleep latency and impairs sleep quality, ${ }^{47-49}$ as the intention to fall asleep fast can generate cognitive arousal which impairs sleep. Conversely, insomnia patients can profit from the paradoxical instruction "to try to stay awake". ${ }^{35}$ In our study, we did not find any evidence for a strong impairing effect of the intention to sleep "good" on sleep in young healthy participants on our primary outcome measure.

However, in our exploratory analysis, during the "sleep good" night the percentage of REM sleep was significantly reduced and occurred descriptively (but not significantly) later, as compared to the other two conditions. The exact role of REM sleep for "good" vs "bad" sleep is not clear. From research in depressive participants, an earlier onset of REM sleep and more REM sleep has been associated with stronger depressive symptoms, and a reduction in
REM sleep has been suspected to be beneficial for treatment outcomes. ${ }^{50-54}$ However, whether less REM sleep is beneficial for emotional regulation in healthy participants is not clear. As participants were also not instructed to manipulate REM sleep, the reason and impact of the reduction of REM sleep after the instruction to sleep "good" remains elusive but may be interpreted as an impairment of sleep.

In contrast to the "good" condition, the instruction to sleep "bad" clearly impaired sleep quality both on the level of subjective but also objective sleep parameters. Thus, healthy participants are able to impair their sleep intentionally. Participants were particularly capable of extending the time it took them to fall asleep. Importantly, they were not allowed to get up, but they had to lie in bed in the darkness without any distraction (eg, reading, video, social media, etc.). Thus, they had to stay awake only using their intention as a way of affecting their sleep. As for the strategies used, they reported keeping their eyes open, actively generating (negative) thoughts and planning activities for the next day. Objectively, by using these strategies they managed to stay awake 17 mins longer on average, compared to the "neutral" night. While this is a robust and reliable extension of SOL by intention, the additional time of $17 \mathrm{mins}$ is also not very much, and an SOL of 30 mins in total is probably still in the normal range. Thus, in healthy sleepers, sleep pressure is strong enough to override intentions and induce sleep within 30-45 mins, although some subjects were able to stay awake for 75 mins.

In addition to SOL, participants instructed to sleep "bad" were capable of increasing the NWAK as well as the number of shifts from any sleep stage to wake. On the other hand, they were not capable of increasing WASO. This finding is particularly important as it shows that the intention to sleep "bad" was still active while the participant was sleeping, resulting in an increased drive to wake up from the sleep state but not to stay awake as much as possible. The fact that one's brain can automatically and gradually prepare to wake up because one was asked to wake up as much as possible during the night, falls in line with an individuals' ability to program their awakenings at a scheduled time (such as in the case of an alarm). This is indicated by increases in plasma concentration of adrenocorticotropin during the sleep period before the expected awakening, although sleep stages remained unchanged in this study. ${ }^{55}$ Thus, in spite of the strongly reduced consciousness during sleep, our intentions and goals, which are activated during wakefulness before going to sleep, 
remain active during the sleeping state and can influence the sleep state itself. In both the "bad" and "good" conditions, sleep should be affected by our intention. Intentions are part of our executive functions, which are mainly located in the prefrontal cortex (PFC). While the neurobiological mechanisms of intentions are not completely clear, it is widely assumed that they are capable of modulating activity and sensitivity in other brain areas (top-down-regulation). For example, the intention to execute motor movements typically alters motor-related brain activity before the actual initiation of the movement. ${ }^{56,57}$ Intentions and plans "to do something" in the future are typically conceptualized as prospective memories. ${ }^{58-60}$ In addition, intentions can remain active over long periods (eg, "go to the post office tomorrow at 3 pm"). These "prospective memories" rely on prefrontal brain areas, but also involve other memoryrelated brain areas, in particular the parietal lobe (eg, precuneus). ${ }^{61}$ Thus, we assume that the formation of the intention "to sleep worse" or "to sleep better" - based on the PFC - will have top-down influences on the balance between wake-promoting arousal systems and sleeppromoting GABAergic inhibitory systems of our brain (ie, flip-flop switch model of sleep and wake-promoting brain regions). ${ }^{62}$ The top-down influence of the intention will be most influential during the phase of falling asleep, because consciousness is still present during this period. However, together with reduced consciousness during the sleep period, the intention might remain active and have a top-down influence on sleep-maintaining systems. Similar to other prospective memories, this continued influence during sleep might involve memory-related brain areas and mechanisms. Our result suggests that the prospective memory system maintains its function also during the sleep state. The intentions set before sleep are continuously maintained and activated in our prospective memory system throughout sleep. Alternatively, one could speculate that future plans set before sleep are stored as memory representations in the brain. As memories have been shown to be reactivated during certain sleep stages, ${ }^{63}$ it might be possible that future plans are also repeatedly and spontaneously reactivated during sleep. For declarative types of memory, evidence indicates that information acquired before sleep is spontaneously and repeatedly reactivated during SWS and possibly also during NREM sleep stage $2 .{ }^{63}$ On a conceptual level, the instruction to sleep "bad" could also be regarded as a new memory acquired before sleep, which might then be reactivated during sleep alongside other memories. When reactivated, this plan to sleep "bad" and "wake up during the night" could affect the ongoing sleep process, thereby resulting in an increased number of awakenings during sleep as well as more shifts from any sleep stage to wake. However, this explanation requires further empirical support.

Generally, our results show that the instructions to sleep "bad" do not only affect SOL but also ongoing sleep processes after sleep onset. These findings are in line with the result that the instruction of an early wakeup time in the morning induces a preparation of a wake response $2-3 \mathrm{hrs}$ before the actual time of awakening. ${ }^{55}$ As follows, this is in consonance with anecdotal reports that people tend to wake up before their alarm clock or hours before an important wake-up time (eg, due to catching a flight).

The effects caused by the instruction to sleep "bad" cannot be explained by increases in physiological arousal before or during sleep. It might have been possible that the participants extended their sleep onset by voluntarily increasing their physiological arousal level while lying in bed. However, heart rate analysis during the period of falling asleep provided no support for this claim. In fact, heart rate even tended to be lower in the "bad" condition as compared to the other two conditions. Thus, a psychological explanation for the decreases in sleep quality is more likely.

The decreased sleep quality after the instruction to sleep "bad" affected behavioral performance in the morning and decreased measures of vigilance. Interestingly, the individual difference in decreased vigilance after the "bad" night could not be explained neither by differences in subjective nor objective sleep parameters. Thus, it remains unclear which sleep parameter exactly contributes to the reduction in vigilance. The significant correlation in the "good" condition raises the suspicion that reaction time in the PVT might be particularly influenced by the subjective evaluation of one's own sleep quality. However, as this correlation is not present in the "bad" condition, the increase in reaction time cannot be explained by participants' impression that they have slept worse.

SOL, WASO and NWAK were generally overestimated in the subjective reports as compared to the objective parameters, but these parameters on the subjective level correlated positively with the same parameters on an objective level, with small to medium effect sizes. Interestingly, the instruction to sleep "bad" caused a higher subjectiveobjective discrepancy as compared to the "good" and "neutral" condition. As scientists are aware of the effects of a reward-related task (ie, receiving money as compensation 
for good performance), one could speculate that participants rated their sleep worse in the "bad" condition because they wanted to fulfill the expectations of the experimenter who asked them to sleep "bad". However, this kind of demand characteristic should equally affect subjective and objective ratings in the "good" condition. Here, no evidence of the same participants fulfilling the experimenter's expectations was detectable in the data. Therefore, we consider the role of demand characteristics for the subjective sleep ratings as minor. Especially because we showed that differences exist between objective and subjective sleep parameters. A higher discrepancy between objective and subjective sleep parameters is an important symptom in subtypes of insomnia patients, ${ }^{12,64}$ and a decrease of this discrepancy is related to improvements in insomnia symptoms. ${ }^{65,66}$ A recent pilot study suggests that the subjective opinion of one's own sleep length and quality can have an even stronger impact on feelings of tiredness and impaired cognitive performance the next day when measured as objective sleep parameters. ${ }^{67}$ Our results show that the simple instruction to sleep "bad" can strongly increase the discrepancy between objective and subjective sleep parameters, while the same person shows a much lower discrepancy when he or she expects his or her sleep to be normal or even good. This finding highlights the possibility that our perception of sleep largely depends on our psychological goals and intentions related to our sleep, and less on changes of objective parameters during sleep (see eg, ${ }^{68}$ ).

While our study clearly shows that intentions are capable of influencing sleep in healthy participants, objective changes in sleep parameters, in particular, remain rather small. For example, we did not observe any effects of the sleep instructions on power in the SWA band, which we already know has been implicated in the process of homeostatic regulation of sleep. This result shows that a major part of objective sleep is regulated by physiological mechanisms that are beyond our intentional control. Our results also suggest that intentional processes cannot explain previous reports on the extension of SWS and the increase in SWA by means of more subconscious interventions (eg, placebo effects, ${ }^{69}$ music, ${ }^{29,70}$ hypnotic suggestions ${ }^{44}$ ). Thus, the sole instruction of sleeping "good", "bad" or "neutral" is not enough to find large effects on objective sleep architecture.

\section{Limitations}

Although our study design was already complex, we are aware that three nights are not sufficient to verify all relevant points of our hypothesis. Among others, one limitation regards our study design: we only used three experimental nights. In particular, an additional night without any instructions would have been important to estimate whether our "neutral" instruction could also alter, or at least influence, sleep. As a second point, the criteria of absence of sleep disorders in our recruiting process (only healthy sleepers are recruited to participate in this non-clinical trial) was only confirmed by the PSQI. ${ }^{19}$ This questionnaire is used as an instrument to assess self-reported sleep quality and does not allow to identify sleep disturbances or insomnia based on proper diagnostic criteria. Additional tests for sleep disorders were not conducted nor was sleep history assessed throughout our participants. However, the very high sleep efficiency renders sleep disorders in our sample rather unlikely. Another limitation of our study is that participants may have reached a ceiling effect in different sleep parameters, such as sleep efficiency, resulting in high values in all conditions. Therefore, discrimination between participants in the different conditions was unfeasible, thus the expected improvement when trying to sleep better than normal was not possible. The lack of changes in different sleep parameters may be due to a partially sleep-restricted population resulting from the instruction to get up at 7 am on the day of the experiment. For higher clinical relevance, future studies should systematically examine the influence of intentions on sleep in participants with sleep disturbances.

\section{Conclusion}

In sum, here we have shown that intentions can impair, but not improve healthy sleep. Thus, bad sleep can be the result of our intention to sleep "bad". Furthermore, the intention to sleep "bad" increases the discrepancy between subjective and objective sleep parameters, resulting in a stronger overestimation of the SOL, WASO and NWAK as compared to the other two instructions. Furthermore, participants were also capable of extending their sleep onset and increasing their NWAK on an objective level. Thus, the goal to sleep "bad" was still active during the sleep state and resulted in a disturbance of the sleep process, suggesting that goals and intentions are relevant for maintaining a stable sleep state. While the simple instruction to sleep "good" was not enough to further improve sleep, recent studies conducted in our laboratory show that the amount of SWS can be extended by using more subconscious techniques, such as hypnotic suggestions. ${ }^{44,45}$ Taken together, our intentions, thoughts 
and imagination are important influencing factors of our subjective and objective sleep quality.

\section{Acknowledgments}

We thank Fiona Hart-Hoenig for leading the pilot study as well as Valentina Arnold, Sarah Locher, Rahel Schärli, and Anna Wick for helping with the data collection. A special thank goes to Louisa Clarke for her effort in proofreading. This study was supported by a grant from the European Research Council (ERC) under the European Union's Horizon 2020 research and innovation program (grant agreement MemoSleep No. 677875).

\section{Disclosure}

Financial disclosure: AVA AG, Zurich supported the project with 900.00 CHF. Non-financial disclosure: The authors report no conflicts of interest for this work.

\section{References}

1. Borbély AA, Achermann P. Sleep homeostasis and models of sleep regulation. Journal of Biological Rhythms. 1999;14(6):559-568. doi:10.1177/074873099129000894

2. Greenblatt DJ, Shader RI. Dependence, tolerance, and addiction to benzodiazepines: clinical and pharmacokinetic considerations. Drug Metab Rev. 1978;8(1):13-28. doi:10.3109/03602537808993775

3. Hindmarch I, Dawson J, Stanley N. A double-blind study in healthy volunteers to assess the effects on sleep of pregabalin compared with alprazolam and placebo. Sleep. 2005;28(2):187-193. doi:10.1093/ sleep/28.2.187

4. Holbrook A, Crowther R, Lotter A, Endeshaw Y. The role of benzodiazepines in the treatment of insomnia: meta-analysis of benzodiazepines in the treatment of insomnia. J Am Geriatr Soc. 2001;49 (6):824-826. doi:10.1046/j.1532-5415.2001.49161.x

5. Roehrs T, Roth T. Sleep, sleepiness, sleep disorders and alcohol use and abuse. Sleep Med Rev. 2001;5(4):287-297. doi:10.1053/ smrv.2001.0162

6. Brunner DP, Dijk DJ, Münch M, Borbély AA. Effect of zolpidem on sleep and sleep EEG spectra in healthy young men. Psychopharmacology. 1991;104(1):1-5. doi:10.1007/BF02244546

7. Lader M. Rebound insomnia and newer hypnotics. Psychopharmacology. 1992;108(3):248-255. doi:10.1007/BF02245108

8. Aeschbach D, Dijk D-J, Trachsel L, Brunner DP, Borbély AA. Dynamics of slow-wave activity and spindle frequency activity in the human sleep EEG: effect of midazolam and zopiclone. Neuropsychopharmacology. 1994;11(4):237-244. doi:10.1038/sj. npp. 1380110

9. Dijk D-J. Regulation and functional correlates of slow wave sleep. J Clin Sleep Med. 2009;5(2 Suppl):6-15. doi:10.5664/jcsm.5.2S.S6

10. Harvey AG, Tang NKY, Browning L. Cognitive approaches to insomnia. Clin Psychol Rev. 2005;25(5):593-611. doi:10.1016/j. cpr.2005.04.005

11. Harvey AG. A cognitive model of insomnia. Behav Res Ther. 2002;40:869-893. doi:10.1016/S0005-7967(01)00061-4

12. Rezaie L, Fobian AD, McCall WV, Khazaie H. Paradoxical insomnia and subjective-objective sleep discrepancy: A review. Sleep Med Rev. 2018;40:196-202. doi:10.1016/j.smrv.2018.01.002
13. Riemann D, Spiegelhalder K, Feige B, et al. The hyperarousal model of insomnia: A review of the concept and its evidence. Sleep Med Rev. 2010;14(1):19-31. doi:10.1016/j.smrv.2009.04.002

14. Riemann D, Baglioni C, Bassetti C, et al. European guideline for the diagnosis and treatment of insomnia. J Sleep Res. 2017;26 (6):675-700. doi:10.1111/jsr.12594

15. Riemann D, Perlis ML. The treatments of chronic insomnia: A review of benzodiazepine receptor agonists and psychological and behavioral therapies. Sleep Med Rev. 2009;13(3):205-214. doi:10.1016/j.smrv.2008.06.001

16. Haynes J-D, Sakai K, Rees G, Gilbert S, Frith C, Passingham RE. Reading Hidden Intentions in the Human Brain. Curr Biol. 2007;17 (4):323-328. doi:10.1016/j.cub.2006.11.072

17. Momennejad I, Haynes J-D. Human anterior prefrontal cortex encodes the 'what' and 'when' of future intentions. Neuroimage. 2012;61(1):139-148. doi:10.1016/j.neuroimage.2012.02.079

18. Griefahn B, Kunemund C, Brode P, Mehnert P. Zur Validitat der deutschen Ubersetzung des Morningness-Eveningness-Questionnaires von Horne und Ostberg. The Validity of a German Version of the Morningness-Eveningness-Questionnaire Developed by Horne and Ostberg. Somnologie. 2001;5(2):71-80. doi:10.1046/j.1439-054X.2001. 01149.x

19. Buysse DJ, Reynolds III CF, Monk TH, Berman SR, Kupfer DJ. The Pittsburgh Sleep Quality Index: A new instrument for psychiatric practice and research. Psychiatry Res. 1989;28(2):193-213. doi:10.1016/0165-1781(89)90047-4

20. Rasch B, Born J, Gais S. Combined blockade of cholinergic receptors shifts the brain from stimulus encoding to memory consolidation. J Cogn Neurosci. 2006;18(5):793-802. doi:10.1162/jocn.2006.18.5.793

21. Oldfield RC. The assessment and analysis of handedness: the Edinburgh inventory. Neuropsychologia. 1971;9(1):97-113. doi:10.1016/0028-3932(71)90067-4

22. Steyer R, Schwenkmezger P, Notz P, Eid M. MDBF - Mehrdimensionaler Befindlichkeitsfragebogen. Hogrefe: Göttingen; 1997.

23. Görtelmeyer R. SF-A/R Und SF-B/R: Schlaffragebogen $A$ Und $B$. Bern: Hogrefe; 2011. http://ub-madoc.bib.uni-mannheim.de/29052.

24. Bongartz W. German Norms for the Harvard Roup Scale of Hypnotic Susceptibility, Form a. International Journal of Clinical and Experimental Hypnosis. 1985;33(2):131-139. doi:10.1080/ 00207148508406643

25. Dinges DF, Powell JW. Microcomputer analyses of performance on a portable, simple visual RT task during sustained operations. Behavior Research Methods, Instruments, \& Computers. 1985;17 (6):652-655. doi:10.3758/BF03200977

26. Iber C, Ancoli-Israel S, Chesson A, Quan SF. For the American Academy of Sleep Medicine. The AASM Manual for the Scoring of Sleep and Associates Events: Rules, Terminology and Technical Specifications. Version 1. 1st ed ed. Westchester, IL: American Academy of Sleep Medicine; 2007.

27. Weber FD SleepTrip - A branch of FieldTrip with added functionality for sleep analyses. 2019. https://github.com/Frederik-D-Weber/sleeptrip.

28. Krystal AD. Non-REM sleep EEG spectral analysis in insomnia. Psychiatr Ann. 2008;38(9):615-620. doi:10.3928/00485713-20080901-08

29. Cordi MJ, Ackermann S, Rasch B. Effects of Relaxing Music on Healthy Sleep. Sci Rep. 2019;9(1):1-9. doi:10.1038/s41598-01945608-y

30. Maes J, Verbraecken J, Willemen M, et al. Sleep misperception, EEG characteristics and Autonomic Nervous System activity in primary insomnia: A retrospective study on polysomnographic data. Int J Psychophysiol. 2014;91(3):163-171. doi:10.1016/j.ijpsycho.2013.10.012

31. Tarvainen MP, Lipponen J, Niskanen J-P, Ranta-aho PO. Kubios HRV Version 3.3: User's Guide. 2019:40.

32. Kogler L, Müller VI, Chang A, et al. Psychosocial versus physiological stress - meta-analyses on deactivations and activations of the neural correlates of stress reactions. Neuroimage. 2015;119:235-251. doi:10.1016/j.neuroimage.2015.06.059 
33. Finelli LA, Baumann H, Borbély AA, Achermann P. Dual electroencephalogram markers of human sleep homeostasis: correlation between theta activity in waking and slow-wave activity in sleep. Neuroscience. 2000;101(3):523-529. doi:10.1016/S0306-4522(00) 00409-7

34. Borbély AA. From slow waves to sleep homeostasis: new perspectives. Arch Ital Biol. 2001;139(1-2):53-61. doi:10.4449/aib. v139i1.204

35. Morin CM, Hauri PJ, Espie CA, Spielman AJ, Buysse DJ, Bootzin RR. Nonpharmacologic treatment of chronic insomnia: an American Academy of Sleep Medicine Review. Sleep. 1999;22 (8):1134-1156. doi:10.1093/sleep/22.8.1134

36. Allada R, Cirelli C, Sehgal A. Molecular mechanisms of sleep homeostasis in flies and mammals. Cold Spring Harb Perspect Biol. 2017;9(8):1-20. doi:10.1101/cshperspect.a027730

37. Nicassio P, Bootzin R. A comparison of progressive relaxation and autogenic training as treatments for insomnia.. Journal of Abnormal Psychology. 1974;83(3):253-260. doi:10.1037/h0036729

38. Means MK, Lichstein KL, Epperson MT, Johnson CT. Relaxation therapy for insomnia: nighttime and day time effects. Behaviour Research and Therapy. 2000;38(7):665-678. doi:10.1016/S00057967(99)00091-1

39. Kahn M, Baker BL, Weiss JM. Treatment of insomnia by relaxation training.. Journal of Abnormal Psychology. 1968;73(6):556-558. doi: $10.1037 / \mathrm{h} 0026599$

40. Winkler A, Rief W. Effect of Placebo Conditions on Polysomnographic Parameters in Primary Insomnia: A Meta-Analysis. Sleep. 2015;38 (6):925-931. doi:10.5665/sleep. 4742

41. Neukirch N, Colagiuri B. The placebo effect, sleep difficulty, and side effects: A balanced placebo model. J Behav Med. 2015;38 (2):273-283. doi:10.1007/s10865-014-9590-5

42. Colagiuri B, McGuinness K, Boakes RA, Butow PN. Warning about side effects can increase their occurrence: an experimental model using placebo treatment for sleep difficulty. J Psychopharmacol. 2012;26(12):1540-1547. doi:10.1177/0269881112458730

43. Lai HL, Good M. Music improves sleep quality in older adults. $J A d v$ Nurs. 2004;53(1):2006. doi:10.1111/j.1365-2648.2006.03693.x

44. Cordi MJ, Schlarb AA, Rasch B. Deepening sleep by hypnotic suggestion. Sleep. 2014;37(6):1143-1152. doi:10.5665/sleep.3778

45. Cordi MJ, Rossier L, Rasch B. Hypnotic Suggestions Given Before Nighttime Sleep Extend Slow-Wave Sleep As Compared To a Control Text in Highly Hypnotizable Subjects. International Journal of Clinical and Experimental Hypnosis. 2020;68 (1):105-129. doi:10.1080/00207144.2020.1687260

46. Bayer L, Constantinescu I, Perrig S, et al. Rocking synchronizes brain waves during a short nap. Curr Biol. 2011;21(12):R461R462. doi:10.1016/j.cub.2011.05.012

47. Espie CA, Wicklow A. Cognitive therapy for insomnia. In: Shapiro C, Sloan EP, editors. Sleep in Psychiatry. Cambridge University Press; 1999.

48. Wicklow A, Espie CA. Intrusive thoughts and their relationship to actigraphic measurement of sleep: towards a cognitive model of insomnia. Behaviour Research and Therapy. 2000;38(7):679-693. doi:10.1016/S0005-7967(99)00136-9

49. Schmidt RE, Courvoisier DS, Cullati S, Kraehenmann R, Linden MVD. Van der Linden M. Too imperfect to fall asleep: perfectionism, pre-sleep counterfactual processing, and insomnia. Front Psychol. 2018;9(AUG):1-11. doi:10.3389/fpsyg.2018. 01288

50. Habukawa M, Uchimura N, Maeda M, Ogi K, Hiejima H, Kakuma T. Differences in rapid eye movement (REM) sleep abnormalities between posttraumatic stress disorder (PTSD) and major depressive disorder patients: REM interruption correlated with nightmare complaints in PTSD. Sleep Med. 2018;43:34-39. doi:10.1016/j. sleep.2017.10.012
51. Pesonen A-K, Gradisar M, Kuula L, et al. REM sleep fragmentation associated with depressive symptoms and genetic risk for depression in a community-based sample of adolescents. J Affect Disord. 2019;245 (August 2018):757-763. doi:10.1016/j.jad.2018.11.077

52. Steiger A, Kimura M. Wake and sleep EEG provide biomarkers in depression. J Psychiatr Res. 2010;44(4):242-252. doi:10.1016/j. jpsychires.2009.08.013

53. Steiger A, Pawlowski M. Depression and sleep. Int J Mol Sci. 2019;20(3):1-14. doi:10.3390/ijms20030607

54. Wichniak A, Riemann D, Kiemen A, Voderholzer U, Jernajczyk W. Comparison between eye movement latency and REM sleep parameters in major depression. Eur Arch Psychiatry Clin Neurosci. 2000;250(1):48-52. doi:10.1007/s004060050009

55. Born J, Hansen K, Marshall L, Mölle M, Fehm HL. Timing the end of nocturnal sleep [6]. Nature. 1999;397(6714):29-30. doi:10.1038/16166

56. Jeannerod M. The representing brain: neural correlates of motor intention and imagery. Behav Brain Sci. 1994;17(2):187-202. doi:10.1017/S0140525X00034026

57. Wairagkar M, Hayashi Y, Nasuto SJ, Sakakibara M. Exploration of neural correlates of movement intention based on characterisation of temporal dependencies in electroencephalography. Sakakibara M, ed. PLoS One. 2018;13(3):e0193722. doi:10.1371/journal.pone.0193722

58. Barner C, Seibold M, Born J, Diekelmann S. Consolidation of prospective memory: effects of sleep on completed and reinstated intentions. Front Psychol. 2017;7(JAN):1-18. doi:10.3389/fpsyg. 2016.02025

59. Barner C, Altgassen M, Born J, Diekelmann S. Effects of sleep on the realization of complex plans. J Sleep Res. 2019;28(1):1-9. doi: $10.1111 /$ jsr. 12655

60. Fabbri M, Tonetti L, Martoni M, Natale V. Sleep and prospective memory. Biological Rhythm Research. 2014;45(1):115-120. doi: $10.1080 / 09291016.2013 .830510$

61. Burgess PW, Gonen-Yaacovi G, Volle E. Functional neuroimaging studies of prospective memory: what have we learnt so far? Neuropsychologia. 2011;49(8):2246-2257. doi:10.1016/j.neuropsychologia.2011.02.014

62. Saper CB, Chou TC, Scammell TE. The sleep switch: hypothalamic control of sleep and wakefulness. Trends Neurosci. 2001;24 (12):726-731. doi:10.1016/S0166-2236(00)02002-6

63. Rasch B, About Sleep's BJ. Role in Memory. Physiol Rev. 2013;93 (2):681-766. doi:10.1152/Physrev.00032.2012

64. Manconi M, Ferri R, Sagrada C, et al. Measuring the error in sleep estimation in normal subjects and in patients with insomnia: insomnia. J Sleep Res. 2010;19(3):478-486. doi:10.1111/j.13652869.2009.00801.x

65. Crönlein T, Wetter TC, Rupprecht R, Spiegelhalder K. Cognitive behavioral treatment for insomnia is equally effective in insomnia patients with objective short and normal sleep duration. Sleep Med. 2020;66:271-275. doi:10.1016/j.sleep.2018.10.038

66. Mitchell LJ, Bisdounis L, Ballesio A, Omlin X, Kyle SD. The impact of cognitive behavioural therapy for insomnia on objective sleep parameters: A meta-analysis and systematic review. Sleep Med Rev. 2019;47:90-102. doi:10.1016/j.smrv.2019.06.002

67. Rahman SA, Rood D, Trent N, Solet J, Langer EJ, Lockley SW. Manipulating sleep duration perception changes cognitive performance - an exploratory analysis. J Psychosom Res. 2020;132 (March):109992. doi:10.1016/j.jpsychores.2020.109992

68. Riemann D, Spiegelhalder K, Nissen C, Hirscher V, Baglioni C, Feige B. REM sleep instability - A new pathway for insomnia? Pharmacopsychiatry. 2012;45(5):167-176. doi:10.1055/s-0031-1299721

69. Fratello F, Curcio G, Ferrara M, et al. Can an inert sleeping pill affect sleep? Effects on polysomnographic, behavioral and subjective measures. Psychopharmacology. 2005;181(4):761-770. doi:10.1007/ s00213-005-0035-2

70. Lai H-L H-L, Good M. Music improves sleep quality in older adults. $J$ Adv Nurs. 2005;49(3):234-244. doi:10.1111/j.1365-2648.2004. 03281.x 


\section{Publish your work in this journal}

Nature and Science of Sleep is an international, peer-reviewed, open access journal covering all aspects of sleep science and sleep medicine, including the neurophysiology and functions of sleep, the genetics of sleep, sleep and society, biological rhythms, dreaming, sleep disorders and therapy, and strategies to optimize healthy sleep.

Submit your manuscript here: https://www.dovepress.com/nature-and-science-of-sleep-journa|
The manuscript management system is completely online and includes a very quick and fair peer-review system, which is all easy to use. Visit http://www.dovepress.com/testimonials.php to read real quotes from published authors. 